\title{
Testing the Empirical Validity of the Adaptive Markets Hypothesis
}

\author{
HANY FAHMY \\ University of Waterloo *
}

The issue of market efficiency attracted the attention of academicians since the existence of financial markets. Over time, two schools of thoughts were established: the efficient markets school and the behavioral finance school. Proponents of the former believed in the Efficient Markets Hypothesis whereas the latter brought evidence from behavioral finance and psychology to demonstrate that financial markets are inefficient and this inefficiency is attributed to irrational behavior of investors in making financial choices regarding asset allocation and portfolio construction. Recently, an adaptive reconciliation was suggested, which posits that investors' adaptability is what brings back inefficient markets to efficiency. The purpose of this paper is to test empirically the validity of the Adaptive Markets Hypothesis via a smooth transition regression model with market-tobook ratio as exogenous threshold variable. The results support the reconciliation and show that markets are indeed efficient sometimes and inefficient most of the time.

Keywords: Efficient Markets Hypothesis, Adaptive Markets Hypothesis, Smooth Transition Regression Models, Regime Switching Models, Irrationality, S\&P500, Marketto-Book Ratio

JEL Classifications: C1, C3.

\section{Introduction}

Academicians have had interests in studying and understanding the behavior of financial time series data since the existence of financial markets. Over time, two schools of thoughts were established: the efficient markets school and the behavioral finance school. Proponents of the former believed in the Efficient Markets Hypothesis (EMH), which posits that capital markets are ideal in the sense that, at any point in time, asset prices in any market fully reflect all available information. The latter school, on the other hand, brought evidence from behavioral finance and psychology showing that investors, especially retail traders, exhibit irrational behavior in making investment decisions, i.e., asset allocation and portfolio construction, which can explain the observed violations of the EMH in capital markets. Andrew Lo (2004) surveyed

*Department of Mathematics; hfahmy@uwaterloo.ca; hfahmy@hf-consulting.ca. The author would like to acknowledge the University of Waterloo Mathematics Endowment Fund for its generous support of this research project.

(C) 2017 Hany Fahmy. Licensed under the Creative Commons Attribution - Noncommercial 3.0 Licence (http://creativecommons.org/licenses/by-nc/3.0/). Available at http: //rofea.org. 
the literature on the debate between the advocates of the EMH and behavioral finance and suggested a reconciliation between both approaches. His reconciliation hypothesis, which he called "the Adaptive Markets Hypothesis (AMH)," posits that market inefficiency is due to irrational behavior of investors. But since investors adapt to the changing environment, their adaptability over time brings the market back to efficiency. Basically, Lo's AMH posits that financial markets transition between episodes of inefficiencies, but the adaptability of its participants continuously forces them to revert back to efficiency. The objective of this paper is to test the empirical validity of the AMH via regime switching models. In particular, we employ the logistic smooth transition regression model of order 1 (LSTR(1)) pioneered by Granger and Teräsvirta (1993) and Teräsvirta (1994). The LSTR(1) model is best suited here for it is capable of describing processes that can move from one regime to another such that the transition is smooth. Since the AMH suggests a switching behavior between episodes of efficiencies and inefficiencies in capital markets, then by applying such a model to a rate of return of a market price index, denoted by $r_{t}$ in the text, one can capture the switching behavior, if it exists, of $r_{t}$ between these episodes. Moreover, the degree of smoothness of this switching behavior can be taken to reflect the speed of the adaptability process, i.e., how fast investors react to financial news; a smooth transition reflects a slower steady adaptability whereas an abrupt transition from one regime to another is indicative of a faster and more swift adaptability to financial news or shocks.

The selection of the transition variable in the LSTR(1) model is crucial as it explains the dynamics of $r_{t}$, i.e., it explains how the market rate of return moves from one regime to another. The convention in the regime switching literature is to use a lag order autoregressive component of the dependent variable, i.e., $r_{t-d}$, where $d$ is a delay parameter, as the transition variable. However, the AMH suggests that the transition variable that governs the regime switching behavior of $r_{t}$ ought to be an exogenous variable that captures investors' reactions to financial news. It turns out that the best exogenous variable that captures the dynamic of $r_{t}$ is the one period lag of the first difference of the market-to-book (M/B) ratio, denoted by $\Delta\left(\frac{M}{B}\right)_{t-1}$ in the text. This makes sense intuitively since all available market information are reflected in the price of the market index, which in turn, is captured by the M/B ratio. Thus, changes in $M / B$ ratio in period $t-1$ have an impact on the market rate of return in period $t$. Our statistical analysis confirms that $\Delta\left(\frac{M}{B}\right)_{t-1}$ is indeed the best transition variable in the fitted STR model that is capable of capturing the dynamic of $r_{t}$.

The paper is organized as follows: Section 2 describes the data set, introduces the STR model, and explains the rationale behind using $\Delta\left(\frac{M}{B}\right)_{t-1}$ as an exogenous threshold variable. Section 3 summarizes the empirical results, and Section 4 concludes. 


\section{FAHMY Testing the Adaptive Market Hypothesis}

\section{Regime Switching in the S\&P500}

The market rate of return studied in this paper is the Standard and Poor (S\&P500) index over the period between the fourth quarter of 1969 and the second quarter of 2014. A plot of the index rate of return, $r_{t}$, which is defined as $r_{t}=\frac{P_{t+1}-P_{t}}{P_{t}}$, where $P_{t}$ is the end-of-quarter closing price of the S\&P500 composite index taken from Compustat and Center of Research on Security Prices (CRSP) merged file data base, is depicted in the top panel of Figure 1. The transition variable is the first difference of the one-period lag of the market-to-book ratio, $\Delta\left(\frac{M}{B}\right)_{t-1}$, which is defined as $\Delta\left(\frac{M}{B}\right)_{t-1}=\left(\frac{M}{B}\right)_{t-1}-\left(\frac{M}{B}\right)_{t-2}$, where $M_{t}$, the market value in period $t$, is computed as the sum of all shares outstanding for asset $i$ times the quarter closing price of asset $i$, for all assets included in the index in period $t$, and $B_{t}$, the book value in period $t$, is the sum of shareholder's equity for all assets included in the index in period $t$. The transition variable $\Delta\left(\frac{M}{B}\right)_{t-1}$ is depicted in the bottom panel of Figure $1 .^{1}$

The rationale behind using the $\mathrm{M} / \mathrm{B}$ ratio as an exogenous transition variable in the STR model is that it is determined by the closing prices of all stocks in its composition. And since stock prices reflect all market information, the $\mathrm{M} / \mathrm{B}$ ratio is an excellent candidate that can capture investors' reactions to financial news as reflected in the continuous changes in stock prices. A quick survey of the literature confirms this rationale: financial ratios that have market prices in their definitions seem to have explanatory power in explaining the variation of the expected rate of return on a market portfolio. For instance, in the context of the Capital Asset Pricing Model (CAPM), Basu (1977) discovered an earning-to-price (E/P) ratio effect. In particular, Basu showed that when common stocks are sorted on E/P, future returns on high E/P stocks are higher than predicted by CAPM. Banz (1981) reported a size effect; when stocks are sorted on market capitalization, average returns on small stocks are higher than predicted by CAPM. Bhandari (1988) reported a leverage effect; high debt-to-equity ratios are associated with returns that are too high relative to their market betas. Statman (1980) and Rosenberg, Reid, and Lanstein (1985) showed that stocks with high book-to-market equity ratios have high average returns than the returns suggested by their beta coefficients. Recently Fama and French (1992, 1993) and Pontiff and Schall (1998) confirmed the ability of the M/B ratio to predict market returns. These authors also acknowledged the predictive ability of other factors such as dividend yields or bond variables, e.g., default spread and yield to default. After experimenting with all the pervious suggested ratios, the statistical and empirical analysis showed that the $M / B$ ratio is the best exogenous transition variable that is capable of capturing the nonlinear (regime

${ }^{1}$ The quarterly data on closing prices, shares outstanding, and shareholder's equity of all underlying constituents of the S\&P500 since 1969 was used to construct the M/B ratio using the following two guidelines: (1) any asset that was not included in the S\&P500 in period $t$, i.e., quarter $t$, was dropped from the data set. (2) Any asset that showed no value for either its stock price or the shareholder's equity or the number of shares outstanding was also dropped from the computations. 
switching) dynamic of the S\&P500 rate of return. In particular, the statistical analysis either didn't reject the null hypothesis of linearity in market return when one of these factors was used as an exogenous threshold variable or rejected the null hypothesis of linearity, but the estimated model didn't pass the diagnostic tests. The model against which the rejection, measured in pvalue, was the strongest was the STR model with M/B ratio as threshold variable.

Figure 1: The quarterly rates of return on the S\&P500 and the first difference of the oneperiod lag of the market-to-book ratio between 1969:Q4 and 2014:Q2.

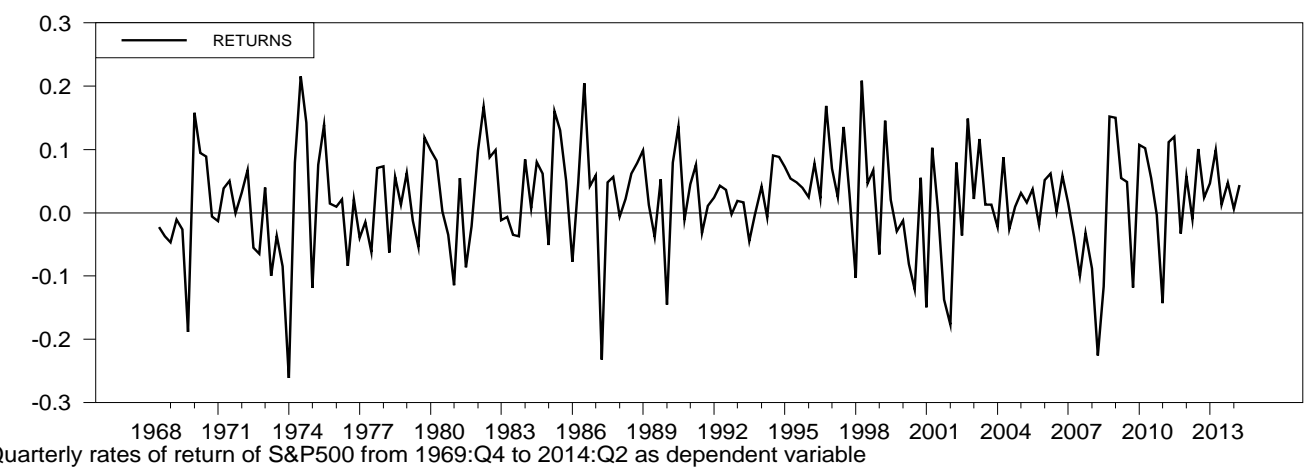
Quarterly rates of return of S\&P500 from 1969:Q4 to 2014:Q2 as dependent variable

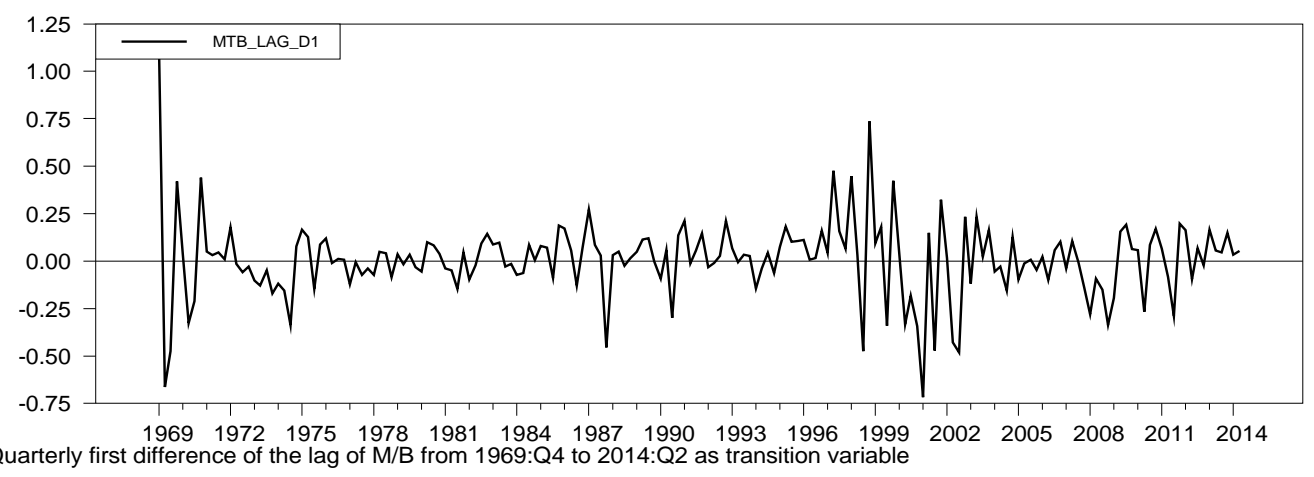

A quick look at Figure 1, one can notice that the transition variable is capable of capturing every bear and bull episode of the S\&P500 over the given period. For instance, the crash of 19691970 that resulted from the drop in consumer confidence as a result of the high inflation and the increased deficits from the Vietnam War is reflected in the two swings in the transition variable, i.e., $\Delta\left(\frac{M}{B}\right)_{t-1}$, during the few quarters between 1969 and 1970. The financial crash of 1974 that followed the OPEC oil embargo in 1973 is well noticed in the downfall of the S\&P500 returns as shown in the upper panel of Figure 1. This swing is also captured by the transition variable in the lower panel. The crash of 1987, which followed the contractionary monetary policy adopted by the Federal Reserve in 1986 to combat inflation, is also picked up by the 
transition variable. The so-called "Dot-Com bubble" effect that caused the clear downfall of the S\&P500 in the second half of 2002 as shown in the upper panel of Figure 1 was also captured by the M/B ratio as shown from the down spike in 2001. The recent Housing Bubble that bursted in 2008 is also clearly captured by the transition variable. The M/B ratio also seems to capture the bull episodes in the S\&P500 quite well, and thus, acts as a good transition variable in the STR model.

Before we proceed to the empirical analysis, a few words regarding the STR model employed in this paper are in order. STR models have been used extensively in the regime switching literature. For a recent review, see Fahmy (2014) and the references therein. ${ }^{2}$

In a compact notation, the standard STR model of order $p$ is expressed as follows.

$$
r_{t}=\boldsymbol{\beta}^{\prime} \boldsymbol{r}_{t}+\boldsymbol{\Theta}^{\prime} \boldsymbol{r}_{t} \mathrm{G}\left(s_{t} ; \gamma, \boldsymbol{c}\right)+\varepsilon_{t}, \quad t=1, \ldots, T,
$$

where $r_{t}$ is the market rate of return at time $t, \boldsymbol{r}_{t}^{\prime}=\left(1, r_{t-1}, \ldots, r_{t-p}\right), \boldsymbol{\beta}^{\prime}=\left(\beta_{0}, \beta_{1}, \ldots, \beta_{p}\right)$, and $\boldsymbol{\Theta}^{\prime}=\left(\theta_{0}, \theta_{1}, \ldots, \theta_{p}\right)$, are parameter vectors, $\varepsilon_{t} \sim$ i.i.d. $\left(0, \sigma^{2}\right)$, and $\mathrm{G}\left(s_{t} ; \gamma, \boldsymbol{c}\right)$ is a bounded (between 0 and 1) transition function of the continuous transition variable $s_{t}$, which will be taken to be $\Delta\left(\frac{M}{B}\right)_{t-1}$, and continuous everywhere in the parameter space for any value of $s_{t}$. The transition function $\mathrm{G}(\cdot)$ is a logistic function defined in general as

$$
\mathrm{G}\left(s_{t} ; \gamma, \boldsymbol{c}\right)=\left(1+\exp \left\{-\gamma \prod_{i=1}^{k}\left(s_{t}-c_{i}\right)\right\}\right)^{-1}, \quad \gamma>0
$$

where $\gamma$ is the slope of the function, and $c=\left(c_{1}, \ldots, c_{k}\right)^{\prime}$ is a vector of location parameters such that $c_{1} \leq c_{2} \leq \cdots \leq c_{k}$. Given such definition, the STR model defined in (1) is then referred to as the logistic smooth transition regression (LSTR) model.

The transition function in (2) is a monotonically increasing function of the transition variable $\mathrm{s}_{t}$. The restrictions $\gamma>0$ and $\mathrm{c}_{1} \leq \mathrm{c}_{2} \leq \cdots \leq \mathrm{c}_{k}$ are identifying restrictions. The choice of $k$ determines the behavior of the logistic transition function. Two common choices for $k$ are used in the literature: $k=1$ and $k=2$. In the LSTR model with $k=1$ (LSTR(1)), the parameters vectors change monotonically as a function of $s_{t}$ from $\boldsymbol{\beta}$ to $\boldsymbol{\beta}+\boldsymbol{\Theta}$. This reflects the capability of the LSTR(1) model to characterize processes whose dynamic properties are different in an upper regime from what they are in a lower regime such that the transition

${ }^{2}$ It is worth mentioning that regime switching can also be modelled in the variance of $r_{t}$; threshold (G)ARCH and STR-(G)ARCH models are good candidates for capturing the asymmetric response of asset returns to external shocks. In the preliminary stage of writing this paper, we experimented with such models. They, however, didn't yield significant estimates. Modelling nonlinearity in the mean equation of $r_{t}$ via STR models was found to be a better alternative. 
between the two regimes is smooth. The LSTR model with $k=2$ give rise to three regimes; two outer regimes and a middle one. Our results show, as we will see shortly, that the logistic function in the fitted STR model to the market rate of return is of order 1 . This means that the market rate of return displays two distinct inefficient regimes; a lower inefficient regime where market price is below its book value (undervalued) and an upper inefficient regime where market price is above its book value (overvalued). But, since the transition from one inefficient regime to another as entailed by the LSTR(1) model suggests that the market index passes briefly by an episode of efficiency where market price is close to its book value, then one can also take this swift transition as indicative of a third efficient regime in between. The market price doesn't, however, persist in this efficient regime due to irrational behavior of market participants (especially retail investors) in response to financial news and other exogenous shocks. This is consistent with the adaptability process entailed by the AMH.

The logistic function of order 1 takes the form

$$
\mathrm{G}\left(s_{t} ; \gamma, c\right)=\left(1+\exp \left\{-\gamma\left(s_{t}-c\right)\right\}\right)^{-1}, \quad \gamma>0
$$

Notice that when $s_{t} \rightarrow-\infty, \mathrm{G}(\cdot)=0$; this defines the lower regime, whereas when $s_{t} \rightarrow+\infty$, $\mathrm{G}(\cdot)=1$ and the time series is said to be in an upper regime.

In order for the series under consideration to be able to fluctuate between the two upper and lower (inefficient) regimes, they need to have some sort of mean reversion mechanism, and

hence, they ought to be stationary. The stationarity of $r_{t}$ and $\Delta\left(\frac{M}{B}\right)_{t-1}$ is confirmed at the $5 \%$ level of significance by the ADF, PP, and KPSS tests as shown from Table 1. This is consistent with our hypothesis that the market rate of return is pushed by changes in its market-to-book value from one inefficient regime to another passing by a brief episode of efficiency.

\section{Empirical Results}

Following the modelling framework proposed by Granger and Teräsvirta (1993), Teräsvirta (1994), and Eitrheim and Teräsvirta (1996), which consists of three stages, we begin by specifying an adequate linear $A R(m)$ model for $r_{t}$, where $\mathrm{m}$ is the value that minimizes the Akaike (1974) information criterion (AIC). Before accepting the suggested $A R(m)$ model as the starting point of the regime switching analysis, preliminary diagnostic tests should be applied to the preliminary model in order to ensure its adequacy as a starting linear model. In particular, the Ljung-Box (1978) test of no serial correlation of order $q=1$ up $q=4$ in the residuals and Engle's (1982) Lagrange multiplier (LM) test of no autoregressive conditional heteroskedasticity (ARCH) of order $v=1$ up to $v=4$ in the residuals are considered. 


\section{FAHMY Testing the Adaptive Market Hypothesis}

Table 1: The stationarity of the Standard and Poor returns and the first difference of the market-to-book ratio over the period between 1969 and 2014.

\begin{tabular}{|c|l|l|l|}
\hline Time Series & ADF $(k)$ & PP & KPSS \\
\hline$r_{t}$ & $-10.56(1)$ & -15.51 & 0.08 \\
\hline$\Delta\left(\frac{M}{B}\right)_{t}$ & $-9.66(1)$ & -12.42 & 0.09 \\
\hline
\end{tabular}

The AIC for $r_{t}$ was minimized at a lag order $m=1$, and therefore, the preliminary linear model for the nonlinearity analysis was an $A R(1)$ model. The results of the estimated preliminary $A R(1)$ model and the previous misspecification tests are summarized as follows:

$$
\begin{gathered}
r_{t}=\underset{(0.007)}{0.02}+\underset{(0.07)}{0.087} y_{t-1}+\hat{a}_{t}, \\
Q(1)=0.96, \quad Q(8)=0.76, \\
L M_{A R C H(1)}=0.21, \quad L M_{A R C H(4)}=0.09, \\
J B=0.08, \quad k_{3}=-0.46, \quad k_{4}=3.64,
\end{gathered}
$$

where $\hat{a}_{t}$ is the series of residuals, $k_{3}$ and $k_{4}$ are skewness and kurtosis respectively, $Q(q)$ is the p-value of the Ljung-Box (1978) test, $J B$ is the p-value of the Jarque-Bera (1987) test of normality, and the figures in parentheses beneath the estimated parameters are the standard errors. Judging by the p-value of the Ljung-Box (1978) test, the null hypothesis of no serial correlation of order $q=1$ up to $q=8$ in the residuals series was not rejected at the $5 \%$ level of significance. Also, judging by the p-value of Engle's (1982) test, the null hypothesis of no ARCH of order $v=1$ up to $v=4$ was not rejected at the $5 \%$ level of significance. Finally, the null hypothesis of normality of errors was not rejected at the $5 \%$ level of significance as seen from the p-value of the $J B$ test. Judging by the previous tests, the $A R(1)$ model passes all preliminary diagnostic tests and can act as a good starting model for the nonlinearity analysis.

The next step in the specification stage is nonlinearity testing. The null hypothesis of linearity, denoted by $H_{0 L}$ in the text, is tested against the alternative of a nonlinear STR model. We shall adopt the nonlinearity test suggested by Teräsvirta (1994) and Luukkonen et al (1988), which is basically an LM test with an asymptotic F distribution when $H_{0 L}$ is valid. The authors also suggested a sequence of $\mathrm{F}$ tests, denoted by $F_{2}, F_{3}$, and $F_{4}$ in the text, to choose an appropriate type of STR model, i.e., LSTR(1) or LSTR(2). In summary, the purpose of nonlinearity testing is twofold: First, it allows the researcher to identify which transition 
variable, in a set of transition candidates, is suitable for the STR model. Second, the F-tests procedure identifies what type of STR model fits the data best, i.e., LSTR(1) or LSTR(2). ${ }^{3}$

The convention in the regime switching literature is to specify a set of candidate transition variables, e.g., time trend, autoregressive lags of the dependent variable, or other sensible exogenous threshold variables, and repeat the nonlinearity test for each transition candidate in the transition set. If the null hypothesis of linearity, $H_{0 L}$, using the $\mathrm{F}$ test, denoted by $F_{L}$ in the text, is rejected for at least one of the models, the model against which the rejection, measured in the p-value, is strongest is chosen to be the STR model to be estimated. Although, our earlier proposition suggested that $\Delta\left(\frac{M}{B}\right)_{t-1}$ is the potential transition variable in the STR model, for the sake of completeness, we performed the nonlinearity test for a time trend, one period lag of $r_{t}$, and for $\Delta\left(\frac{M}{B}\right)_{t-1}$. The model against which the rejection was strongest was the STR model with $\Delta\left(\frac{M}{B}\right)_{t-1}$ as threshold variable and the selected STR model was the LSTR(1) model as shown from the nonlinearity tests results in Table 2.

After determining the transition variable and the type of the STR model, the next step is estimation. The parameters of the STR model in (1) are estimated using conditional maximum likelihood. The log-likelihood function of the STR model is

$l(\boldsymbol{\beta}, \boldsymbol{\Theta}, \sigma, \gamma, c)=-\frac{1}{2} \ln (2 \pi)-\frac{1}{2} \ln \left(\sigma^{2}\right)-\frac{\left\{r_{t}-\left(\boldsymbol{\beta}^{\prime}+\boldsymbol{\Theta}^{\prime} \mathrm{G}\left(s_{t} ; \gamma, c\right)\right) \boldsymbol{r}_{t}\right\}^{2}}{2 \sigma^{2}}$.

Conditional on starting values of the parameters, the log-likelihood function in (4) is maximized using the iterative Broyden-Fletcher-Goldfarb-shanno (BFGS) algorithm. Finding good starting values is important for the algorithm to work properly

Table 2: P-values of the linearity F-tests sequence applied to the Standard and Poor oneperiod lag rate of return, a time trend, and the first difference of the one-period lag market-to-book ratio as exogenous transition variables.

\begin{tabular}{|c|c|c|c|c|l|}
\hline$s_{t}$ & $F_{L}$ & $F_{4}$ & $F_{3}$ & $F_{2}$ & $\begin{array}{l}\text { Suggested } \\
\text { Model }\end{array}$ \\
\hline$r_{t-1}$ & $9.51 \times 10^{-2}$ & $4.24 \times 10^{-1}$ & $5.71 \times 10^{-1}$ & $1.97 \times 10^{-2}$ & Linear \\
\hline Trend & $9.78 \times 10^{-1}$ & $6.92 \times 10^{-1}$ & $8.27 \times 10^{-1}$ & $9.76 \times 10^{-1}$ & Linear \\
\hline$\Delta\left(\frac{M}{B}\right)_{t-1}$ & $9.67 \times 10^{-3}$ & $1.81 \times 10^{-2}$ & $3.64 \times 10^{-2}$ & $3.37 \times 10^{-1}$ & LSTR(1) \\
\hline
\end{tabular}

${ }^{3}$ The nonlinearity test for the STR model and the selection criterion are described in detail in Teräsvirta (1994). 


\section{FAHMY Testing the Adaptive Market Hypothesis}

Starting values are obtained by constructing a grid in $\gamma$ and $c$, estimating the parameter vectors $\boldsymbol{\beta}$ and $\boldsymbol{\Theta}$ conditionally on $(\gamma, c)$ for $k=1$ (LSTR(1) model), and computing the sum of squared residuals. The parameter values that correspond to the minimum of that sum are taken as the starting values. ${ }^{4}$

The last stage in the modelling cycle is the diagnostic stage, where the adequacy of the fitted model is considered. The misspecification tests for the STR models that have been considered in Eitrheim and Teräsvirta (1996) and Teräsvirta (1998) will be considered in this paper. In particular, three tests will be considered. The first test is an LM-type test of no error autocorrelation of order $v$. The p-value of the test is denoted by $L M_{A U T O(v)}$. The second diagnostic test is Engle's (1982) Lagrange multiplier (LM) test of no autoregressive conditional heteroskedasticity (ARCH) of order $q=1$ up to $q=4$ in the residuals. The last diagnostic test is a parameter constancy test, where the null hypothesis of parameter constancy in the STR model is tested against non-monotonic change, non-monotonic symmetrical change, and nonmonotonic and non-symmetrical change with p-values denoted by $P C 1, P C 2$, and $P C 3$ respectively.

The fitted LSTR(1) model and the results of Eitrheim and Teräsvirta (1996) and Teräsvirta’s (1988) misspecification tests are reported as follows:

$$
\begin{gathered}
r_{t}=\underset{(0.098)}{0.32} r_{t-1}+\left(\underset{(0.009)}{0.034}-\underset{(0.15)}{0.54} r_{t-1}\right)\left(1+\exp \left\{-\underset{(702)}{241}\left(\frac{\left.\left.\left.\Delta\left(\frac{M}{B}\right)_{t-1}-\underset{(0.0034)}{0.03}\right)\right\}\right)^{-1}+\hat{\varepsilon}_{t}}{0.18}\right)\right.\right. \\
\hat{\sigma}_{\mathrm{M} / \mathrm{B}}=0.18, \quad \hat{\sigma}=0.08 \\
L M_{A R C H(1)}=0.16, \quad L M_{A R C H(4)}=0.06 \\
L M_{A U T O(1)}=0.72, \quad L M_{A U T O(4)}=0.94, \quad L M_{A U T O(8)}=0.89 \\
J B=0.051, \quad K_{3}=-0.34, \quad K_{4}=3.58 \\
P C(1)=0.10, \quad P C(2)=0.05, \quad P C(3)=0.06
\end{gathered}
$$

where $\hat{\sigma}_{\mathrm{M} / \mathrm{B}}$ is the sample standard deviation of the transition variable $s_{t}=\Delta\left(\frac{M}{B}\right)_{t-1}$ and $\hat{\sigma}$ is the residual standard deviation. The figures in parentheses beneath the parameter values are

4 To facilitate the construction of an effective grid, we follow Teräsvirta's (1998) suggestion to standardize the exponent of the transition function $G\left(s_{t} ; \gamma, c\right)$ by dividing it by the $k^{\text {th }}$ power of the sample standard deviation of the transition variable. This is done mainly to render the parameter $\gamma$ scalefree. 
standard errors. $L M_{A U T O(v)}$ is the p-value for the $v$ th order autocorrelation. $L M_{A R C H(q)}$ is the pvalue of the $q$ th order ARCH. $P C 1, P C 2$, and $P C 3$ are p-values for parameter constancy tests against monotonic change, non-monotonic symmetrical change, and non-monotonic and nonsymmetrical change respectively. The original and fitted series from the STR model of $r_{t}$ are plotted in Figure 2.

Perhaps the most noticeable detail of equation (5) is the large standard deviation of the estimated slope of the logistic transition function $\gamma=241$. It is common for LSTR models that the estimated standard deviation of $\gamma$ tends to be large for large values of $\gamma$. This is not crucial, however, as it does not affect either the shape of the logistic function $G\left(\Delta\left(\frac{M}{B}\right)_{t-1} ; \gamma, c\right)$ or the other estimates of the model. Teräsvirta (1994) gave an example of this exact case and provided a discussion on the estimation issues of $\gamma$. The large slope of the transition function, as depicted from the dot plot in Figure 3, where each dot corresponds to one observation, is indicative of a fast transition of $r_{t}$ from one inefficient regime to another. This is consistent with the AMH, which posits that market participants adapt to changing market conditions. The results also hint to a swift adaptability process; that is, the S\&P500 returns series continuously fluctuates from an upper inefficient regime to a lower inefficient regime passing quickly by an efficient regime. Hence, the market is efficient sometimes but inefficient most of the times.

Figure 2: Original and fitted values of $r_{t}$ between 1969:Q4 and 2014:Q2 with the first difference of the one-period lag of the market-to-book ratio as transition variable.

Plot of Time Series $1970.1-2014.2, T=178$

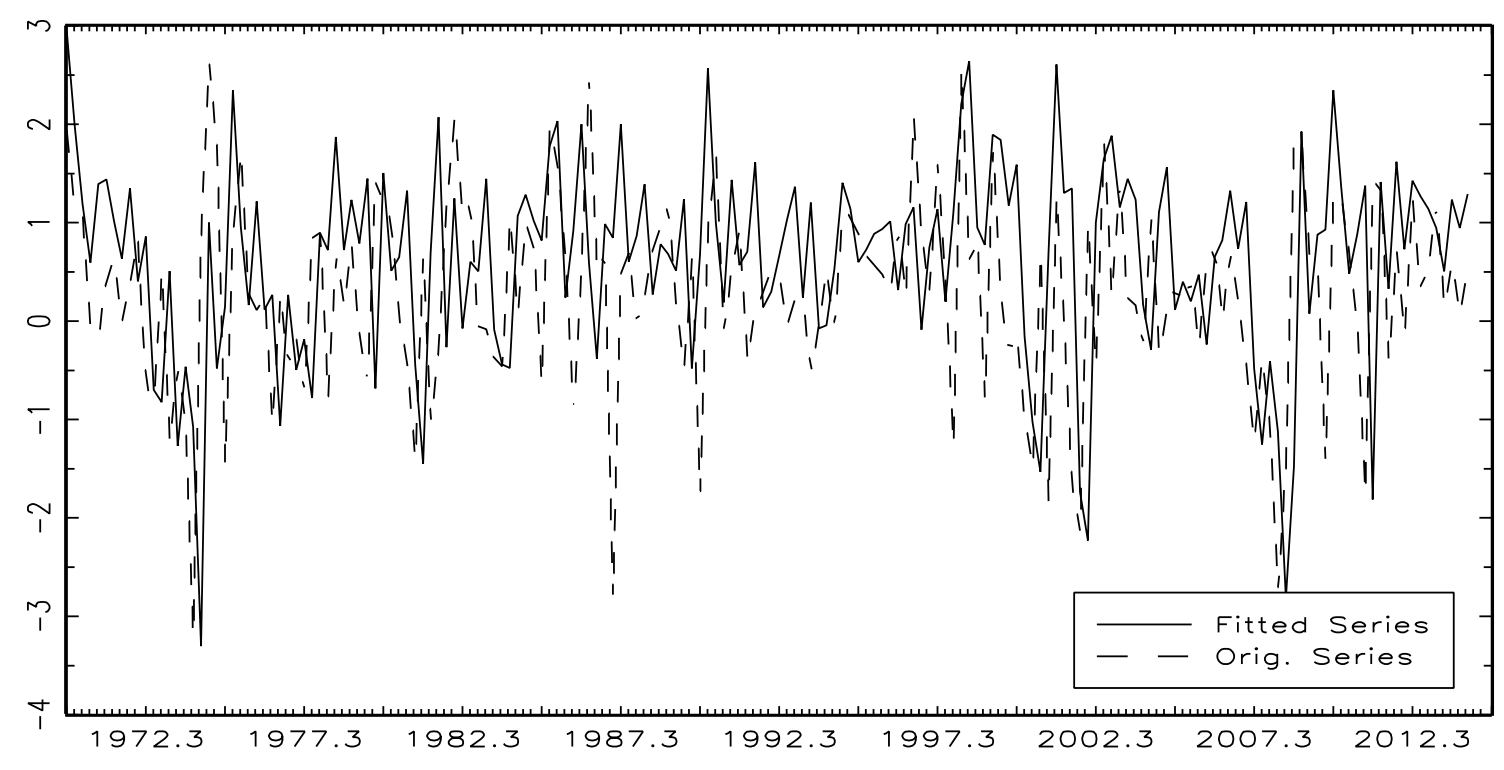




\section{FAHMY Testing the Adaptive Market Hypothesis}

Figure 3: The transition function $\mathrm{G}\left(\Delta\left(\frac{M}{B}\right)_{t-1} ; \gamma, c\right)$ as a function of observations. Each dot corresponds to an observation. The transition variable is the first difference of the one-period lag market-to-book ratio.

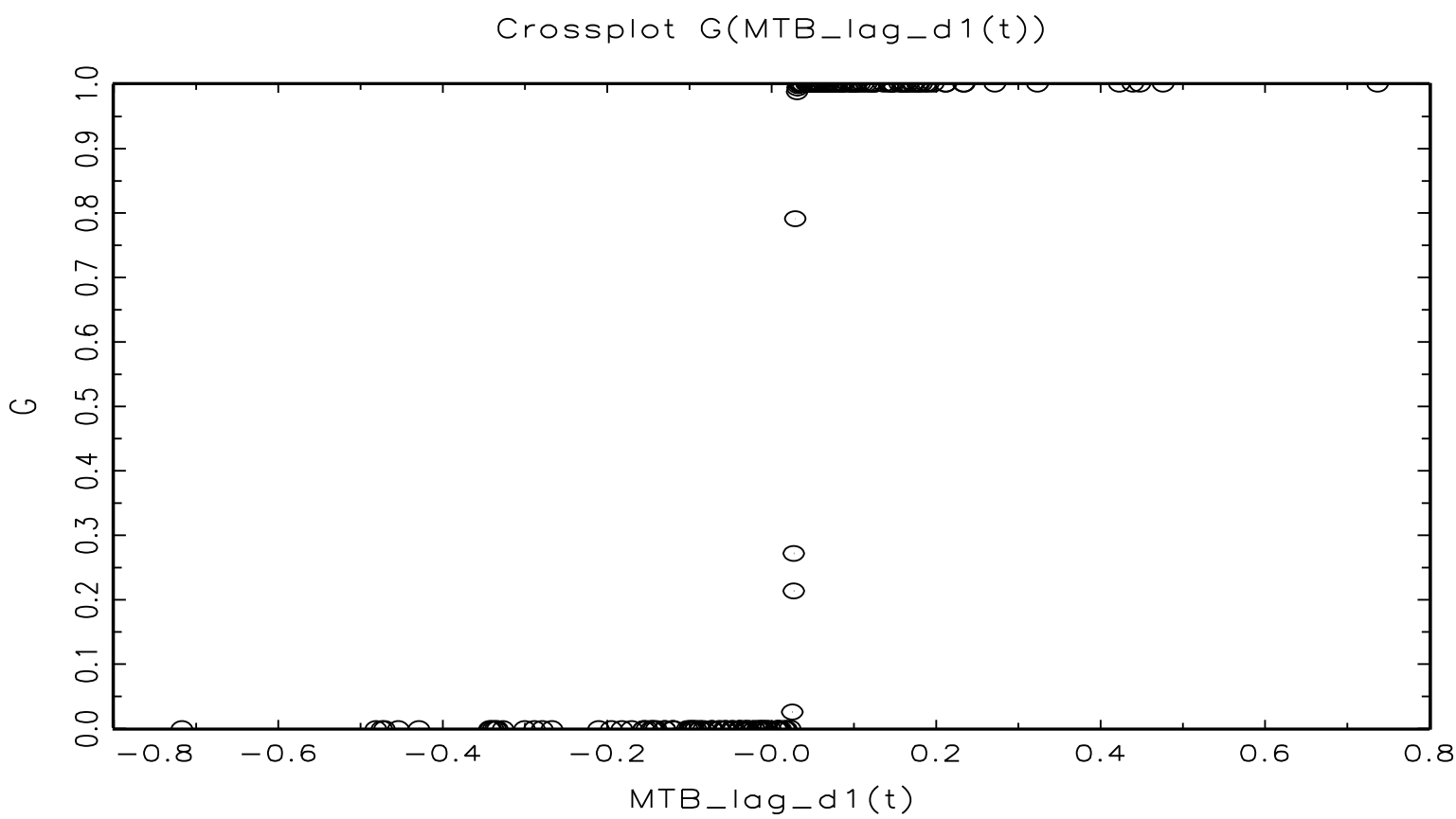

Other than the large slope of the transition function, the estimated coefficients are all significant and the model passes the misspecification tests. One exception is the almost rejection of the null-hypothesis of normality of errors at the $5 \%$ level of significance as seen from the p-value of the JB test statistic. But, this is due to the large outlier of the 1987 crash, where the absolute of the standardized residuals is greater than three as seen from the plot of the standardized residuals in Figure 4.

The dynamic behavior of the market rate of return can be detected by examining the transition function and the behavior of the transition variable above and below the estimated threshold value $\hat{c}=0.03$, which is approximately equal to zero. This means that if the transition variable $\Delta\left(\frac{M}{B}\right)_{t-1}>0.03$, or equivalently, if approximately $\left(\frac{M}{B}\right)_{t-1}>\left(\frac{M}{B}\right)_{t-2}$; that is, if asset prices are overvalued, then the transition function $G(\cdot)$ will approach 1 and the market rate of return will move to an upper stationary inefficient $A R(1)$ regime such that

$$
r_{t}=0.034-0.22 r_{t-1}+\hat{\varepsilon}_{t}
$$


Figure 4: The standardized residuals of the LSTR(1) model in the period between 1969 and 2014 with the first difference of the one-period lag market-to-book ratio.

Plot of Time Series 1970.1-2014.2, $T=178$

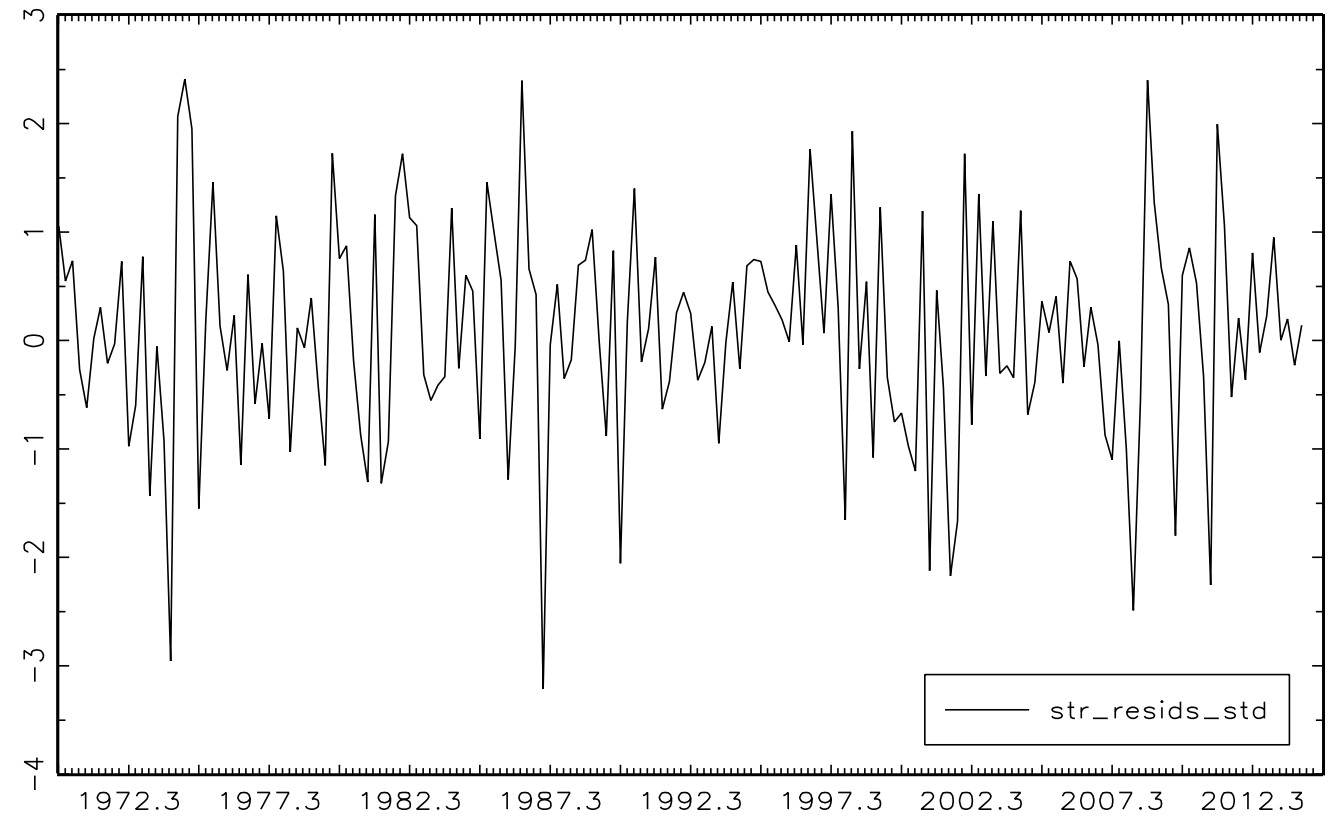

as demonstrated in Figure 5 where the estimated threshold value of 0.03 is the doted line in the upper panel of the figure. On the other hand, if the transition variable is below 0.03 , or approximately, if $\left(\frac{M}{B}\right)_{t-1}<\left(\frac{M}{B}\right)_{t-2}$; that is, if asset prices are undervalued, then the transition function $G(\cdot)$ will approach 0 and the market rate of return will move to a lower stationary inefficient $A R(1)$ regime such that

$$
r_{t}=0.32 r_{t-1}+\hat{\varepsilon}_{t}
$$

The behavior of the S\&P500 rate of return in both inefficient regimes is summarized in Table 3. A simulation of both regimes is also given in Figure 6. Notice that the market rate of return times series wanders in both regimes but always revert back to the mean because the series is stationary. Notice also that the efficient regime is embedded in between the upper and lower inefficient regimes because of the swift swings from one inefficient regime to the other. 
FAHMY Testing the Adaptive Market Hypothesis

Figure 5: A plot of the transition variable $\Delta(M / B)_{t-1}$ and the transition function $\mathrm{G}\left(\Delta(M / B)_{t-1} ; \gamma, c\right)$ between 1969 and 2014.

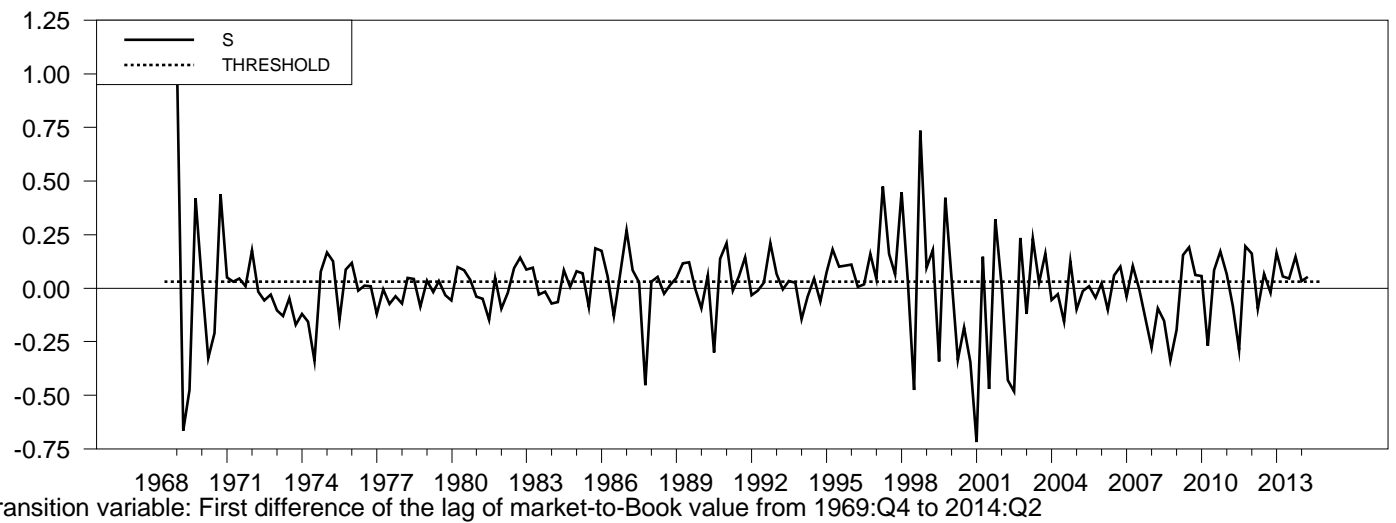

Transition variable: First difference of the lag of market-to-Book value from 1969:Q4 to 2014:Q2

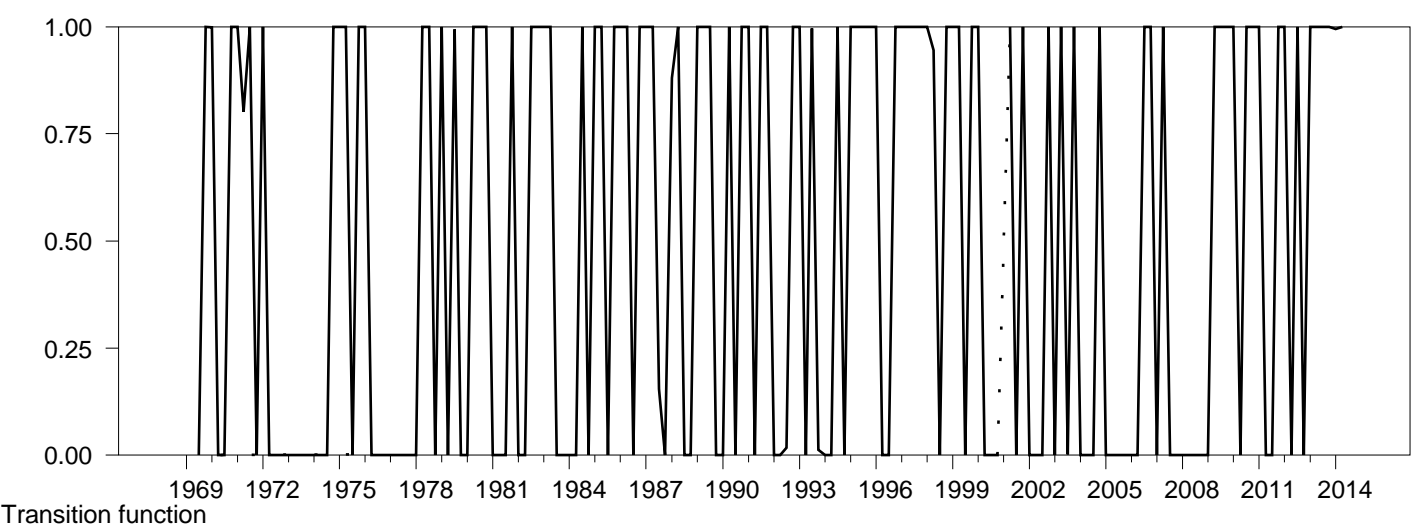

Table 3: The upper and lower regimes of the LSTR(1) model fitted to the rates of return of the Standard and Poor with the first difference of the one-period lag market-to-book ratio as exogenous transition variable.

\begin{tabular}{|l|c|c|}
\hline \multicolumn{1}{|c|}{$s_{t}=\Delta\left(\frac{M}{B}\right)_{t-1}$} & Upper regime: $G(\cdot)=1$ & Lower regime: $G(\cdot)=0$ \\
\hline Threshold: $\hat{c}$ & 0.03 & 0.03 \\
\hline Model & $r_{t}=0.034-0.22 r_{t-1}+\hat{\varepsilon}_{t}$ & $r_{t}=0.32 r_{t-1}+\hat{\varepsilon}_{t}$ \\
\hline Behavior of $r_{t}$ & Stationary AR(1) & Stationary AR(1) \\
\hline Mean & $E\left(r_{t}\right)=0.0278$ & $E\left(r_{t}\right)=0$ \\
\hline Variance & $\operatorname{var}\left(r_{t}\right)=0.0036$ & $\operatorname{var}\left(r_{t}\right)=0.007$ \\
\hline
\end{tabular}


Figure 6: Simulation of the upper $A R(1)$ stationary regime of the quarterly S\&P returns, $r_{t}=0.034-0.22 r_{t-1}+\hat{\varepsilon}_{t}$, and the lower $A R(1)$ stationary regime, $r_{t}=0.32 r_{t-1}+\hat{\varepsilon}_{t}$, based on 250 random realizations.
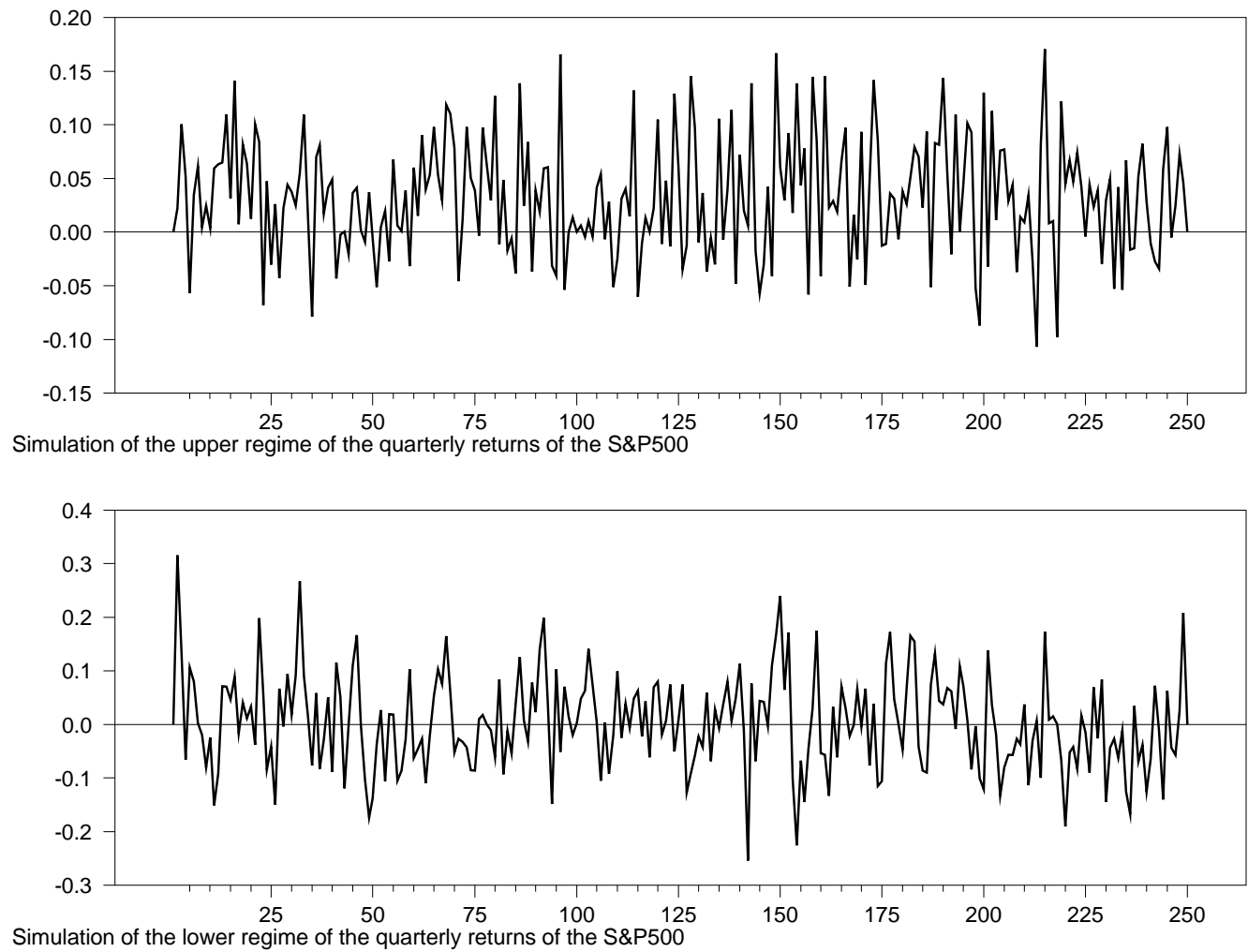

\section{Concluding Remarks}

In this paper, we test empirically the validity of the AMH using a logistic smooth transition regression model of order 1 . The fitted model shows that the rate of return on the S\&P500 index continuously switches from a lower inefficient regime where asset prices are undervalued to an upper inefficient regime where asset prices are overvalued, and in between these two inefficient regimes the market rate of return passes briefly by an episode of efficiency, where its market value is equal to its book value. In summary, the empirical analysis confirms four main implications of the AMH: (1) The adaptability process as suggested by the AMH implies that the two inefficient regimes are both stationary. Otherwise, the market rate of return can wander in one regime without ever returning back to the other. According to our results, both upper and lower (inefficient) regimes were stationary. (2) The adaptability process entails a switching behavior between inefficient regimes. This implies that the market rate of return time series is non-linear and is best modelled by a regime switching model. Our nonlinearity test and the 


\section{FAHMY Testing the Adaptive Market Hypothesis}

regime switching framework confirm that. (3) The transition of the market rate of return from one inefficient regime to another over time is the result of an observed exogenous and stationary factor that is capable of capturing the irrational behavior of market participants; namely, the first difference of the one-period lag market-to-book ratio. The nonlinearity tests sequence and the stationary tests, respectively, confirm that. (4) Finally, the transition between the two inefficient regimes could be smooth or swift depending on the adaptability process of investors. This is also consistent with Lo's AMH, which posits that financial markets witness episodes of inefficiencies, but the adaptability of its participants forces them to revert back to efficiency.

\section{References}

Akaike, H. (1974), A new look at the statistical model identification, IEEE Transactions on Automatic Control 19, 716-723.

Banz, Rolf W. (1981), The Relationship between return and market value of common stocks, Journal of Financial Economics 9, 3-18.

Basu, S. (1977), Investment performance of common stocks in relation to their price-earnings ratios: a test of the efficient market hypothesis, The Journal of Finance 12, 129-56.

Bhandari, Laxmi C. (1988), Debt/Equity ratio and expected common stock returns: empirical evidence, Journal of Finance 43, 507-28.

Dickey, David A. and Fuller, Wayne A. (1979), Distributions of the estimators for autoregressive time series with a unit root, Journal of The American Statistical Association 74, 427-431.

Eitrheim, $\varnothing$. and Teräsvirta, T. (1996), Testing the adequacy of smooth transition autoregressive models, Journal of Econometrics 74, 59-75.

Engle, Robert F. (1982), Autoregressive conditional heteroscedasticity with estimates of the variance of United Kingdom inflation, Econometrica 50, 987-1007.

Fahmy, H. (2014), Modelling nonlinearities in commodity prices using smooth transition regression models with exogenous transition variables, Journal of Statistical Methods and Applications 23, 577-600.

Fama, Eugene F. and French, Kenneth R. (1992), The cross-section of expected stock returns, The Journal of Finance 47, 427-465.

Fama, Eugene F. and French, Kenneth R. (1993), common risk factors in the returns on stocks and bonds, Journal of Financial Economics 33, 3-56.

Granger, C. W. J. and Teräsvirta, T. (1993), Modelling nonlinear economic relationships, Oxford University Press: Oxford.

Jarque, Carlos M. and Bera, Anil K. (1987), A test for normality of observations and regression residuals, International Statistical Review 55, 163-172. 
Kwiatkowski, D., Phillips, Peter C.B., Schmidt, P., and Shin, Y. (1992), Testing the null hypothesis of stationary against the alternative of a unit root: How sure are we that economic time series have a unit root? Journal of Econometrics 54, 159-178.

Lin, C.-F. J. and Teräsvirta, T. (1994), Testing parameter constancy in linear models against stochastic stationary parameters, Journal of Econometrics 90, 193-213.

Ljung, G. M. and Box, G. E. P. (1978), On a measure of lack of fit in time-series models, Biometrika 65, 297-303.

Lo, Andrew W. (2004), The adaptive markets hypothesis: market efficiency from an evolutionary perspective, Journal of Portfolio Management 30, 15-29.

Luukkonen, R., Saikkonen, P., and Teräsvirta, T. (1988), Testing linearity against smooth transition autoregressive models, Biometrika 75, 491-499.

Phillips, P.C.B. and Perron, P. (1988), Testing for a unit root in time series regression, Biometrika 75, 335-346.

Pontiff, J. and Schall Lawrence D. (1998), Book-to-Market ratios as predictors of market returns, Journal of Financial Economics 49, 141-160.

Rosenberg, B., Reid, K., \& Lanstein, R. (1985), Persuasive evidence of market inefficiency, Journal of Portfolio Management 11, 9-17.

Stattman, D. (1980), Book values and stock returns, The Chicago MBA: A Journal of Selected Papers 4, 25-45.

Teräsvirta, T. (1994), Specification, estimation, and evaluation of smooth transition autoregressive models, Journal of American Statistical Association 89, 208-218.

Teräsvirta, T. (1998), Modeling economic relationships with smooth transition regressions. In Handbook of Applied Economic Statistics, Marcel Dekker: New York, 507-552. 